\title{
On the Design of a Remotely-Deployed Detection System for Reactor Assessment at Fukushima Daiichi
}

\author{
Ashley R. Jones, Arron Griffiths, Malcolm J. Joyce, Member, IEEE, Barry Lennox, Senior Member, IEEE,
} Simon Watson, Member, IEEE, Jun-ichi Katakura, Keisuke Okumura, Kangsoo Kim, Michio Katoh, Kazuya Nishimura and Ken-ichi Sawada

\begin{abstract}
The premise behind this research is the design of a system that will allow fuel debris characterisation at Fukushima Daiichi. The precise location of the debris is not known for example as to whether it remains within the reactor pressure vessel or it has leaked through into the base of the pedestal below, additionally the state of the fuel is also in question as to whether this has corroded from within its encasing or if it is intact. The most likely scenario is a combination of all four of these situations. The flooding of the reactor floors immediately following the Fukushima accident adds an extra element of complexity for the detection system requiring it to be submersible and to hold any detector system in water tight confinement. The research carried out has involved extensive modifications to a previously-designed low-cost small-scale AVEXIS submersible inspection vehicle and the incorporation of a variety of radiation detectors. The latter has been designed to allow for mapping and determination of the situation that is present within the primary containment vessels. The challenges addressed with the detection system arise from the high dose rates that have been recorded around the reactor pressure vessels which can be as high as $1000 \mathrm{~Gy} / \mathrm{hr}$. In such a harsh environment not only will the radiation detectors struggle to operate but the components that make up the remote-operated vehicle are also likely to suffer radiation damage after only a relatively short period of time. The research presented here evaluates the components currently incorporated into the AVEXIS system in terms of their radiation tolerability as well as presenting the combination of detectors to be used in the remote probe for the investigation of the fuel debris.
\end{abstract}

\section{INTRODUCTION}

$\mathrm{T}_{\mathrm{H}}$ his paper describes the development of a system comprising a number of detectors to provide information on the status of fuel within the reactors at the Fukushima

This work was funded by the Engineering and Physical and Physical Sciences Research Council (EPSRC: EP/N017749/1) and MEXT (Japan). M. J. Joyce and A. Jones are with the Engineering Department, Lancaster University, UK (telephone: +44 1524 593812, e-mail: m.joyce@lancaster.ac.uk)

A. Griffiths, S. Watson and B. Lennox are with University of Manchester, UK

J. Katakura is with Nagoaka University of Technology, Japan $\mathrm{K}$. Okumura is with the Japan Atomic Energy Agency, Japan

K. Kim, M. Katoh, K. Nishimura and K. Sawada are with the National Maritime Research Institute, Japan
Daiichi nuclear power station in Japan. The environment associated with the fuel is poorly understood however it is hypothesized that in some cases the majority of the fuel debris may have leaked out of the base of the reactor pressure vessel (RPV) into the pedestal below. The pedestal in each of the reactors at Fukushima is submerged to varying depths of water. Currently, estimates of the dose rates inside the primary containment vessel (PCV) vary widely depending on the reactor in question and the exact location of core debris within the PCV. Our target of the highest dose rate is of the order of $100 \mathrm{~Gy} / \mathrm{hr}$ with the objective that inspection instrumentation survive at least 10 hours constituting a requirement that the system withstand a maximum limit of $1 \mathrm{kGy}$ total dose.

\section{AVEXIS SYSTEM}

The inspection and characterization of submerged nuclear environments is a challenging problem. Whilst some facilities are readily accessible and allow the use of traditional remotely-operated vehicles (ROVs), many have very limited access ports in the order range of about $100 \mathrm{~mm}$ diameter. There are few commercial systems which can be deployed through these ports to perform inspection missions.

The Aqua Vehicle Explorer for In-situ Sensing (AVEXIS) [1, 2] vehicles are a range of low-cost, smallscale inspection vehicles that can be deployed through restricted access points. They were originally developed to inspect legacy nuclear fuel storage ponds at the Sellafield nuclear reprocessing site in Cumbria in the United Kingdom. Using novel 3D printing techniques and off-theshelf-components, flexible and low-cost vehicles can be constructed.

The AVEXIS MiniROV (Fig. 1) is $145 \mathrm{~mm}$ in diameter and $250 \mathrm{~mm}$ in length and is designed for the pipework specifically for the legacy storage ponds. It utilizes water pumps located within its end caps for propulsion and carries a high-definition camera for visual inspection. Based on this design, the AVEXIS MicroROV (Fig. 1) has been developed specifically to inspect the PCVs at Fukushima Daiichi.

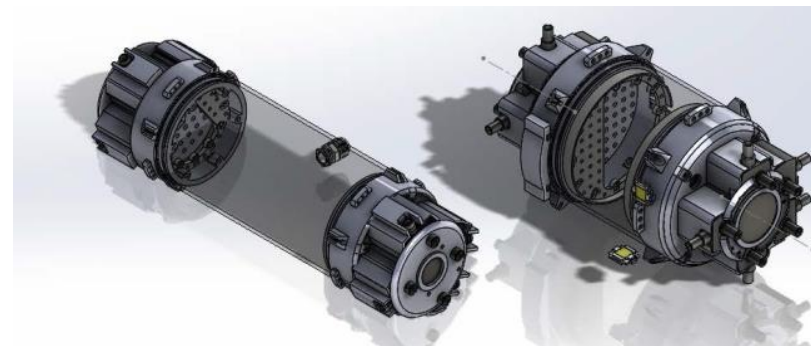

Fig. 1. AVEXIS MicroROV (left) and AVEXIS MiniROV (right). 
The MicroROV is $95 \mathrm{~mm}$ in diameter and $450 \mathrm{~mm}$ in length and has 5 degrees of freedom (DOF). This allows it to be deployed through the existing penetrations in Fukushima Unit 1 (X-100B). It has been designed to carry a payload of detectors for radiation information and a HD colour camera with LEDs for visual feedback and illumination.

The devices within the ROV are powered and accessed remotely using a multi-core tether. For safety reasons it is desirable for the high-voltage supply to any detectors to be produced from within the water tight hull, as opposed to being fed along the tether itself. Taking this in mind the smallest HV supply unit available is a C10940 [3] module supplied by Hamamatsu Photonics K.K. and measures 15 x 15 x $18 \mathrm{~mm}$.

Currently the AVEXIS MicroROV is undergoing gamma irradiation similar to that which is described below for the detectors. This is to ascertain how the components will cope within the high fields associate within the reactors at Fukushima Daiichi. The initial testing involved exposure to the water pumps that will be located at each end of the ROV to varying dose rates and finding the average dose rate failure. The current of each pump was measured during exposure as the indicator of when damage started to occur. The results from these experiments showed an average total dose failure of $\sim 685 \mathrm{~Gy}$, with a minimum total dose failure of $600 \mathrm{~Gy}$. It is presumed that the Arduino microcontroller board could be the first component to suffer radiation damage, this and other important components are currently undergoing testing. After the results of these studies, shielding can be added to protect the more vital components.

\section{DETECTOR PAYLOAD SELECTION}

Prototype payloads consist of one assembly of detectors however two full assemblies will be used in the full system. The two detector assemblies have been designed to allow for an assessment of directionality of both $\gamma$ and neutron fields. The choice of detectors for the each payload assembly was greatly restricted by the radiation levels that would be observed. Although in terms of sensitivity due to the device being a smaller size it offers the benefits of preventing the detector hitting saturation point as easily from such high levels of radiation, the device will also be susceptible to radiation damage. Detectors in widespread use for mixedfield assay at lower dose levels such as organic liquid scintillators were initially investigated for use. These organic liquid scintillators would make ideal detectors due to their ability to discern between different radiation types.

Initial testing utilised a detector comprising of the liquid organic scintillator EJ-301 [4] coupled to a R-5611 photomultiplier tube (PMT) by Hamamatsu Photonics K.K. [5]. The detector is $35 \mathrm{~mm}$ in diameter by $80 \mathrm{~mm}$ in height making it ideal to fit inside a small scale submersible ROV. These tests were undertaken at the University of Manchester's Dalton Cumbria Facility (DCF). At DCF a ${ }^{60} \mathrm{Co}$ irradiator, developed and installed by Foss Therapy Services, INC. [6] was utilised to expose the detector to a gamma only field of equal dose rate to that to be expected within the reactors at Fukushima Daiichi NPS. The ${ }^{60} \mathrm{Co}$ irradiator is a self-shielded device that has three source ports currently comprising of two ${ }^{60} \mathrm{Co}$ sources. These sources can be used in isolation or conjunction with each other to control the dose rate, as well as the chamber allowing the incorporation of attenuation plates to further manipulate the dose. The irradiator is capable of supplying absorbed dose rates between $240-27,000 \mathrm{~Gy} / \mathrm{hr}$.

The initial tests set the detector in a configuration to experience a dose rate of $\sim 950 \mathrm{~Gy} / \mathrm{hr}$ with the expectation that the signal throughput would degrade as saturation was reached. However, the result from this test was instant saturation when the sources were exposed. Due to this outcome the dose rate in the irradiator chamber was reduced gradually until reaching the lowest obtainable dose rate within the chamber of $9.8 \mathrm{~Gy} / \mathrm{hr}$. At this lowest dose rate the detector was on the verge of saturation, delivering data and operating for the first 6-10 seconds of exposure over multiple runs. However, the positive result from these exposures is the detector was exposed to a total absorbed dose of just short of $0.8 \mathrm{kGy}$ showing no sign of radiation damage. The suggestion from these tests was that the detector was on the limit of its ability in terms of saturation at $\sim 10 \mathrm{~Gy} / \mathrm{hr}$, however the material and detector set up does not exhibit any radiation damage in a gamma only field up to the value of $0.8 \mathrm{kGy}$.

From these tests it was decided to move away from liquid organic scintillators due to the inherent experiment safety issues associated with them and the saturation of the amplification electronics meaning they are unsuitable for high flux environments. Similarly newer solid plastic organic scintillators have been discounted not only due to concerns of saturation but also due to the limited radiation dose they can receive before discoloration ensues and opacity develops. Having discounted organic scintillators it was decided that a selection of detectors would need to be used consisting of some neutron sensitive and some gamma sensitive to constitute one payload assembly.

The $\gamma$-ray detector selected incorporated an inorganic cerium bromide $\left(\mathrm{CeBr}_{3}\right)$ crystal of small form factor (the crystal measuring only $10 \mathrm{~mm} \times 10 \mathrm{~mm}$ ). Cerium bromide is a desirable scintillation crystal for nuclear physics due to its fast response and high light yield $[7,8]$ as well as the fast response it exhibits. $[9,10]$. Research has been reported in which a cerium bromide detector was exposed to a ${ }^{60} \mathrm{Co}$ irradiator, in which the photoelectron yield dropped only by $8 \%$ after $111 \mathrm{kGy}$ dose [11]. This surpasses the requirement of surviving to a total dose of 10 kGy.

The $\mathrm{CeBr}_{3}$ crystal [12] is coupled to a $\mathrm{R} 9880-110$ PMT [13] with an inbuilt E-10679-50 [14] voltage divider and C10940 high voltage supply all supplied by Hamamatsu Photonics K.K., into a VS-0087-50 detector configuration supplied by Scionix. This complete detector measures $25 \mathrm{~mm}$ diameter by $100 \mathrm{~mm}$ in length and was specifically designed for use within the ROV in that the submerged tether does not have to carry a high voltage supply reducing the safety risk associated with it. 


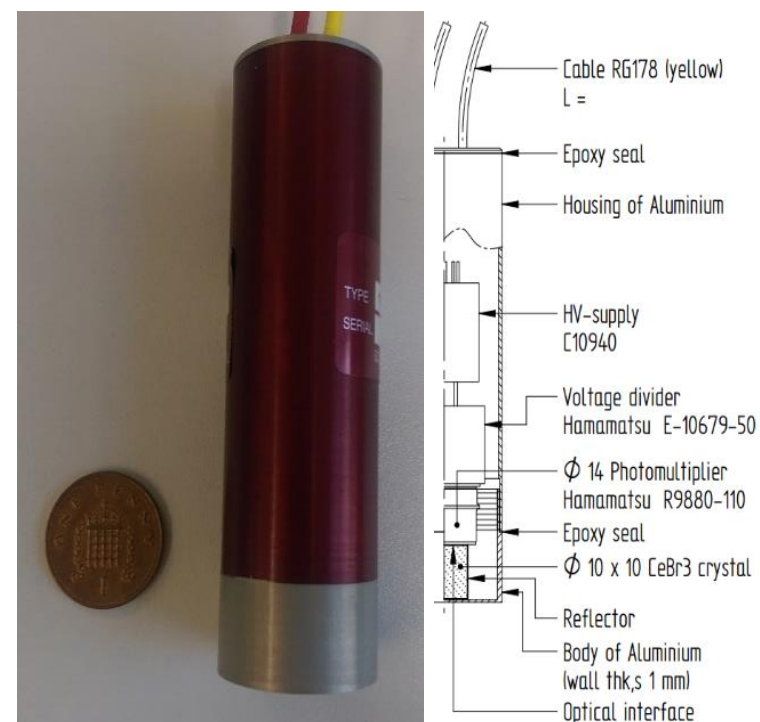

Fig. 2. $\mathrm{CeBr}_{3}$ inorganic scintillator detector, model VS-0087-50 supplied by Scionix, Netherlands [15]

The detector was calibrated using sealed gamma sources, namely ${ }^{137} \mathrm{Cs}$ and ${ }^{22} \mathrm{Na}$, giving the MCA plots shown in Figure 3. From these it is distinguishable that the Compton edges corresponding to the photopeaks of $662 \mathrm{keV}$ and $511 \mathrm{keV}$ for the ${ }^{137} \mathrm{Cs}$ and ${ }^{22} \mathrm{Na}$ sources respectively are visible and consistent between the two spectrums.
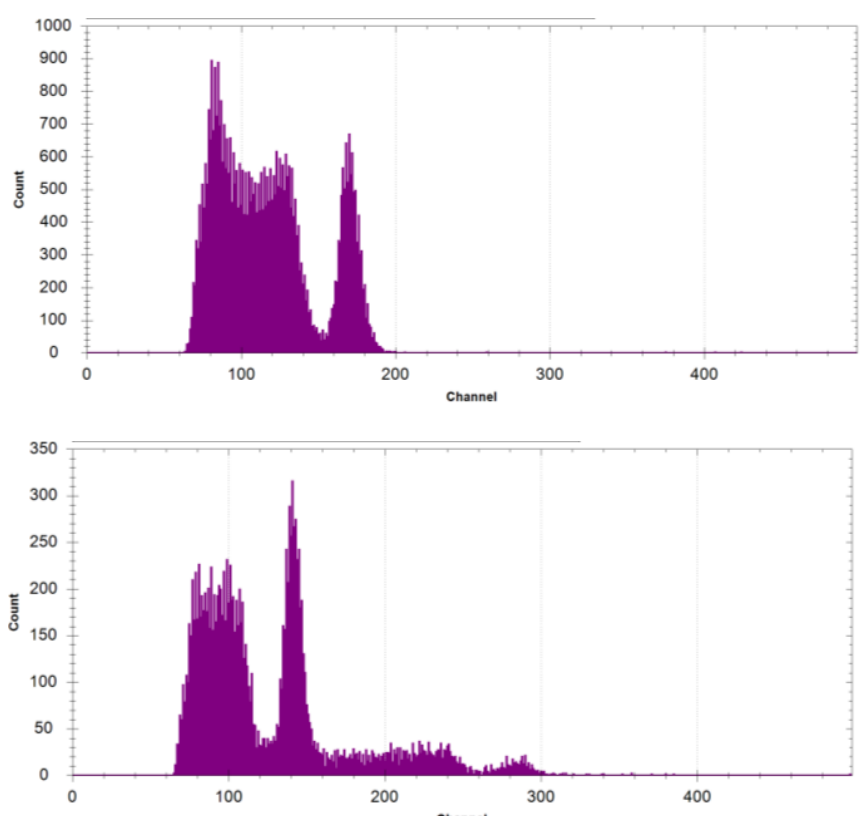

Fig. 3. MCA plots from the $\mathrm{CeBr}_{3}$ inorganic scintillator detector exposed to ${ }^{137} \mathrm{Cs}$ (upper), and ${ }^{22} \mathrm{Na}$ (bottom).

The next stage of investigation for the $\mathrm{CeBr}_{3}$ detector is to incorporate it inside the ROV for wet tests determining dose rates from these sources. For added safety of the detector currently a second layer of containment has been added inside the main hull as a backup for leak purposes. This additional containment consists of a water proof box with water resistant connectors. Wet tests with the detector situated in this second containment hull inside the ROV are set to be undertaken in the near future.

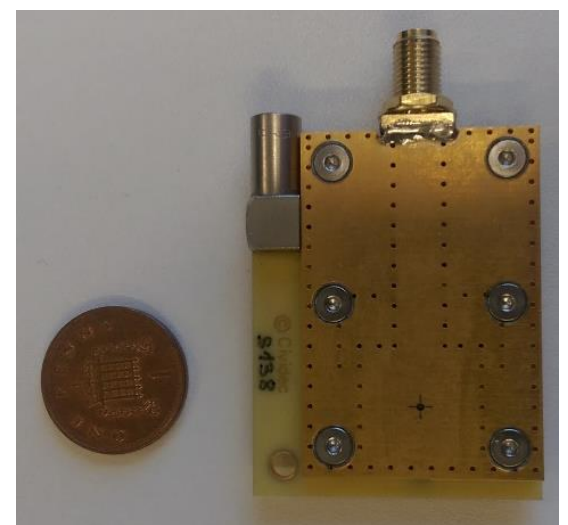

Fig. 4. scCVD thermal-neutron diamond detector, B6, supplied by Cividec Instrumentation, Austria [16].

The neutron detector that was chosen for investigation was a single crystal chemical vapour deposition (scCVD) diamond detector [16], this is shown in Figure 4 . The scCVD has a ${ }^{6} \mathrm{Li}$ foil to increase the efficiency of the detector with respect to the thermal field of neutrons. It is presumed that any fast neutrons in the environment will be slowed by the moderating effects of the water that is submerging the lower plenum of each primary containment vessel.

The prototype payload assembly will consists of one of each of the above detectors, a CAD model of these situated inside the ROV can be seen in Figure 5. This proof of principle prototype will only have directionality from one location to another and will require knowledge of its exact location and each examination point with respect to the previous. The idea is that if successful there could be multiple payload assemblies, one at each end of the central cylindrical body to allow for some source localisation between them. This however will not be implemented in the prototype.

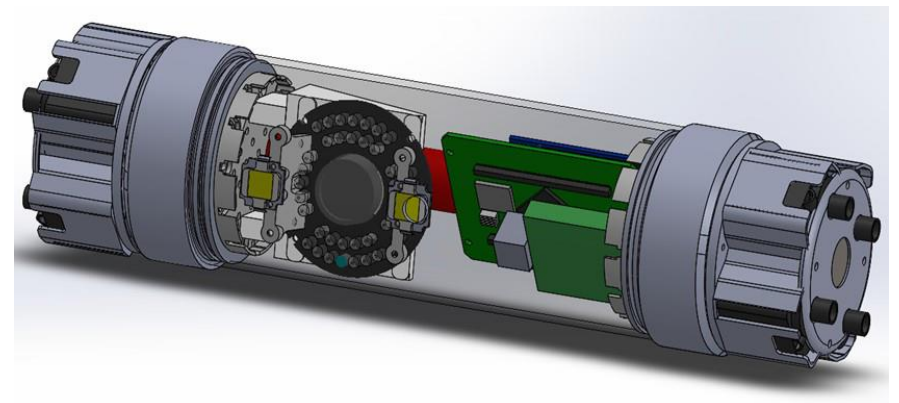

Fig. 5. Detector systems situated inside the AVEXIS MicroROV

\section{CONCLUSIONS}

The research is looking at the investigation of the lower plenum of the reactor units at Fukushima Daiichi NPS. A previously designed submersible small scale ROV has been upgraded and developed from the AVEXIS MiniROV into the MicroROV incarnation. This is fully supporting of the incorporation of at present two detectors, one of these being the $\mathrm{CeBr}_{3}$ and the other being the scCVD diamond detector as described. The detectors that have been described are scheduled for wet testing within the ROV system. These tests involve the deployment of the ROV within a wave tank with gamma sources placed on the outside of the tank for the vehicle to try and identify and locate. 


\section{REFERENCES}

1. S. Watson, A. Stancu \& B. Lennox, "Robotic systems for remote characterisation and decommissioning", Waste Management Symposium Journal, 1(2), (2016)

2. A. Griffiths, et al., "AVEXIS - Aqua Vehicle Explorer for In-Situ Sensing", IEEE Robotics and Automation Letters Vol. 1, (2016)

3. Hamamatsu Photonics K.K. Accessed 25/07/2016 http://www.hamamatsu.com/us/en/product/category/3100/3004/3055/C109 40-53-R2/index.html

4. Eljen Technologies, Accessed 25/07/2016 http://www.eljentechnology.com/index.php/products/liquid-scintillators/ej301-ej-309

5. Hamamatsu Photonics K.K. Accessed 25/07/2016 http://www.hamamatsu.com/eu/en/product/category/3100/3001/R5611A/in dex.html

6. Foss Therapy Services, Accessed 25/07/2016 http://www.fosstherapyservices.net/id12.html

7. S.Ra, et al., "Luminiscence and scintillation properties of a $\mathrm{CeBr}_{3}$ single crystal", IEEE Transactions on Nuclear Science, 55, pp. 1221-1224 (2008)

8. K.S. Shah, et al., " $\mathrm{CeBr}_{3}$ scintillators for gamma-ray spectroscopy", IEEE Transations on Nuclear Science, 52, pp. 3157-3159 (2005)

9. R. Billnert, et al., "New information on the characteristics of 1 in. $x 1$ in. cerium bromide scintillation detectors", Nucl. Instrum. Methods A, 647, pp. 94-99 (2011)

10. N. D'Olympia, et al., "Sub-nanosecond nuclear half-life and time-offlight measurements with $\mathrm{CeBr}_{3}$ ", Nucl. Instrum. Methods A, 728, pp.3135 (2013)

11. W. Drozdowski, et al., " $\mathrm{CeBr}_{3}$ scintillator developed for possible use in space missions" IEEE Trans Nuclear Science, 55, pp. 1391-1396, (2008)

12. John Caunt Scientific Accessed 14/11/2016, http://www.johncaunt.com/detectors/cerium-bromide/

13. Hamamatsu Photonics K.K. Accessed 14/11/2016, http://www.hamamatsu.com/us/en/product/category/3100/3001/R9880U110/index.htm

14. Hamamatsu Photonics K.K. Accessed 14/11/2016 http://www.hamamatsu.com/resources/pdf/etd/E10679_TACC1051E.pdf

15. Scionix NL Accessed 14/11/2016 http://www.scionix.nl/

16. CiviDec Instrumentation Accessed 14/11/2016

https://cividec.at/files/21_pdf.pdf 